Macedonian Pharmaceutical Bulletin, 66 (Suppl 1) 169 - 170 (2020)

Online ISSN $1857-8969$

UDC: 615.212.3.065:[005.52:005.334

DOI: 10.33320/maced.pharm.bull.2020.66.03.084

Short communication

\title{
Implementation of the new EMA methodology of defining risks and the HaRP methodology in the EU Risk Management Plan for Ibuprofen $40 \mathrm{mg} / \mathrm{mL}$ oral suspension
}

\author{
Vladimir Kostovski*, Irina Dukovska, Ivona Trpenoska Aleksovska, Ace Kuzmanovski, \\ Sofija Dimkoska, Marija Crcarovska, Natasha Vukikjevikj
}

Global Pharmacovigilance Unit, Alkaloid AD, Blvd. A.Makedonski 12, 1000 Skopje, North Macedonia

\section{Introduction}

Risk is associated with every single aspect in pharmaceuticals from development of a molecule to its way to the customer (Trivedi et al., 2016). The purpose of the risk management plan (RMP) is to document the risk management system, which is a series of pharmacological activities for identification of the risk, its assessment, minimization or prevention and its communication (Garlapati and Nagandla, 2015). In EEA submission of RMP is required at the time of application for a marketing authorization of a medicine and additionally whenever information affecting the benefit-risk balance of a medicine is available.

After releasing the majority of Good Pharmacovigilance Practices (GVP) guidelines in 2012, The European Medicines Agency (EMA) committed to continuously improve the PV legislation (von Bruchhausen and Schirp, 2017). In early 2016, EMA started a major revision process of the good pharmacovigilance practice (GVP) Module $\mathrm{V}$ on risk management systems.

The final guidance, released at the end of March 2017, set a new milestone in the process of continuous improvement of the RMP guidance. The second revision of GVP module $\mathrm{V}$ provided guidance on the reconsideration of safety concerns from the existing RMP in the post-authorization period (EMA, GVP V, 2017). This encouraged marketing authorization holders (MAHs) to critically revise the list of safety concerns during preparation of the RMP, pharmacovigilance activities, and risk minimization measures in the post-marketing phase.

Revision 2 clarifies that risks should be identified through adverse clinical outcomes that are caused by the use of a medicinal product (identified risks) or that might be caused by the use of a medicinal product (potential risks). The important potential risks are those, when further characterised and if confirmed, would have an impact on the riskbenefit balance of the medicinal product (von Bruchhausen and Schirp, 2017). Important potential risks can be removed from the RMP when they are not being considered important or when there is no reasonable expectation that any pharmacovigilance activity can further characterize the risk, or they can be reclassified to important identified risks.

\section{Materials and methods}

Methodology of HaRP (Harmonisation of RMP Project)

The Coordination Group for Mutual Recognition and Decentralised Procedures - Human (CMDh) in June 2019 have published the document Annex 2:

*vkostovski@alkaloid.com.mk 
HaRP, which aim was to harmonise the RMPs of the same active substances for which marketing authorisations have already been granted with different RMPs in place. First review and harmonisation of the list of safety concerns is performed by the HaRP peer review group and is based on those RMPs published on the Excel list.

Following this initial review, in a subsequent consultation round, feedback is asked from Pharmaceutical Industry and all Member States. The active harmonization can be implemented in two domains. Domain 1 - RMPs for which data exclusivity of reference product will expire. In this domain, the RMP of the reference product will be assessed in the context of revision 2 of GVP Module $\mathrm{V}$ and post-marketing experience with the product to date. Domain 2 referred to clean-up of the List of safety concerns as published on the CMDh website (first step for substances with no reference product or with reference product without a RMP in place). In domain 2, an additional algorithm has been agreed to harmonise the list of safety concerns. For active substances for which there is no innovator or the innovator has no RMP, only those safety concerns should be listed that either have: ongoing additional pharmacovigilance activity, ongoing additional risk minimisation measure, or essential targeted questionnaires in place. In July 2019, CMDh published HaRP assessment reports for twenty-one medicines (CMDh, HaRP, 2019). The new methodology of defining risks and the principles from the HaRP methodology were implemented during preparation of the EU RMP for Ibuprofen $40 \mathrm{mg} / \mathrm{ml}$ oral suspension.

\section{Results and discussion}

The active substance of the medicinal products Ibuprofen $40 \mathrm{mg} / \mathrm{mL}$ oral suspension, (ibuprofen), falls under Domain 2. The proposed important identified risks for ibuprofen in the initial version of the RMP were: gastrointestinal bleeding, cardiovascular events, hepatic and renal disorders, use during pregnancy and severe skin reactions; important potential risk was impaired fertility and proposed missing information were breast feeding and use in children under 6 months of age and under $7 \mathrm{~kg}$ body weight.

Ibuprofen is well known active substance and the safety concerns are already known to the healthcare professionals and adequately described in the product information. For all safety concerns for ibuprofen according to the HaRP methodology there were no ongoing additional pharmacovigilance activity, ongoing additional risk minimisation measures and no essential targeted questionnaires in place. Therefore, in the final version of the document according to the new methodology and the algorithm, all safety concerns were deleted and the summary of safety concerns was published without risks. This approach and the document itself were accepted by the dedicated regulatory agencies in EU, thus the procedure ended with positive outcome.

\section{Conclusion}

RMP is dynamic document that is continually updated throughout the life cycle of the product and when significant information is available. Revision 2 of GVP module $\mathrm{V}$ resulted with simplification and shortening of the Risk management plan (von Bruchhausen and Schirp, 2017), which is also result of using the HaRP methodology, as was the case with the medical writing of RMP for Ibuprofen 40 $\mathrm{mg} / \mathrm{mL}$ oral suspension. The focus of the revised RMP is on identifying or characterizing the safety profile of the medicinal product, proposing measures to prevent or minimize the risks, and including an assessment of the effectiveness of the proposed measures. Expectation is that over time the additional pharmacovigilance activities in a certain RMP in which they are included, will be completed and thus removed from the RMP, or the additional risk minimization activities will be evaluated and modified if necessary throughout the whole life cycle of the product.

\section{References}

Bruchhausen von, T., Schirp, S., 2017. EMA releases the revised Good Pharmacovigilance Practices Module V updated guidance on risk management plans. Medical Writing 26(3), 48-51.

CMDh, 2019. Annex 2: HaRP methodology.

EMA, 2017. Guideline on good pharmacovigilance practices (GVP) Module V - Risk management systems, Rev $2 *$.

Garlapati, S., Nagandla, S., 2015. Risk management plan its importance and emphasys on pharmacovigilance activities. Adv. Pharmacoepidemiol. Drug Saf. 4, 1.

Trivedi, S.R., Shah, B.D., Maheshwari, G.D., 2016. Study on regulatory requirements of risk management plan for pharmaceuticals in Europe, U.S. and Brazil. Indo Global Journal of Pharmaceutical Sciences 6(2), 4958.

Maced. Pharm. Bull. 66 (Suppl 1) 169 - 170 (2020) 